\title{
Optimization, Characterization and in vivo Evaluation of Paclitaxel-Loaded Folate-Conjugated Superparamagnetic Iron Oxide Nanoparticles
}

This article was published in the following Dove Press journal: International Journal of Nanomedicine

\author{
Gang Gui $\mathbb{D}^{1, *}$ \\ Ziqi Fan ${ }^{1} *$ \\ Yihuan Ning' \\ Cancan Yuan ${ }^{2}$ \\ Baolin Zhang (D) ${ }^{3}$ \\ Qin Xu' \\ 'College of Pharmacy, Guilin Medical \\ University, Guilin, 541004, People's \\ Republic of China; ${ }^{2}$ Department of \\ Pharmacy, Luohe City People's Hospital, \\ Luohe, 462000, People's Republic of \\ China; ${ }^{3}$ College of Materials Science and \\ Engineering, Guilin University of \\ Technology, Guilin, 541004, People's \\ Republic of China \\ *These authors contributed equally to \\ this work
}

Background: Paclitaxel (PTX) has interesting anticancer activity. However, it is insoluble in water, which seriously hinders its use in clinical. Superparamagnetic iron oxide nanoparticles (SPIONs) are used as an ideal drug delivery system. Therefore, we proposed a folic acid (FA) targeting drug-loaded SPIONs to reduce its adverse reaction.

Methods: To improve the hydrophilicity of PTX, the structure of PTX was modified by succinic anhydride to obtain 2'-succinate paclitaxel (SPTX). FA conjugated Polyethylene glycol (PEG)/ polyethyleneimine (PEI)-SPIONs SPTX-loaded nanoparticles (SPTX@FA@PEG/PEI-SPIONs) were prepared by solvent volatilization and hydrogen bond adsorption, and the nano-formulation was optimized by response surface methodology (RSM). The characteristics, antitumor effect in vitro, pharmacokinetics, and biodistribution of SPTX@FA@PEG/PEI-SPIONs were evaluated.

Results: SPTX was successfully loaded on the surface of FA@PEG/PEI-SPIONs. The formation of SPTX@FA@PEG/PEI-SPIONs was exhibited water-dispersive monodispersity with high stability by RSM, and dynamic light scattering (DLS) was $178.1 \pm 3.12 \mathrm{~nm}$, particle size observed in the transmission electron microscope (TEM) was $13.01 \pm 1.10 \mathrm{~nm}$, and the encapsulation efficiency (EE) and loading efficiency (LE) were $81.1 \pm 1.66 \%$ and $14.8 \pm 1.46 \%$, respectively. It enhanced the stability in normal physiological condition, accelerated drug release at tumorous $\mathrm{pH}$, and preferentially prolonged the circulation time. In vitro, the SPTX@FA@PEG/PEI-SPIONs significantly targeted to folate receptor (FR) positive cancers cell (HNE-1) via the receptor-ligand mediated pathway, resulting in effective cytotoxic activity. Pharmacokinetic results demonstrated that SPTX@FA@PEG/PEI-SPIONs $\left(\mathrm{t}_{1 / 2}=3.41 \mathrm{~h}\right) \mathrm{had}$ longer than free SPTX or PTX $\left(\mathrm{t}_{1 / 2}=1.67 \mathrm{~h}\right)$ in rats in vivo. Tissue distribution studies showed that SPTX@FA@PEG/PEI-SPIONs were present at high levels in the liver and help in targeting the folate receptors present on the kidneys.

Conclusion: These results suggest that SPTX@FA@PEG/PEI-SPIONs offer a highly promising approach to control drug release, improve drug pharmacokinetics and actively target the nasopharyngeal carcinoma.

Keywords: paclitaxel, folic acid, superparamagnetic iron oxide nanoparticles, targeted drug delivery, response surface methodology, anti-nasopharyngeal carcinoma

\section{Introduction}

Nasopharyngeal carcinoma (NPC) is one of the malignant tumors of nasopharyngeal epithelium in the head and neck, which is particularly prevalent in East and Southeast Asia. ${ }^{1}$ Concurrently, chemoradiotherapy has become the standard treatment for locally advanced nasopharyngeal carcinoma. ${ }^{2,3}$ However, most patients

Baolin Zhang

College of Materials Science and Engineering, Guilin University of Technology, Guilin, 541004, People's Republic of China

Email zhangbaolin@glut.edu.cn 
exhibit resistance to radiotherapy and chemotherapy, resulting in treatment failure. Therefore, it is essential to identify a new targeted drug to reduce NPC chemotherapy resistance to improve treatment effect.

Paclitaxel (PTX), as a first-line chemotherapy option, is used in the treatment of lung cancer, breast cancer, head and neck malignancies and other cancers. ${ }^{2,4,5}$ PTX exhibits good efficacy in patients with NPC during the early stages of treatment, but the subsequent development of resistance is the main cause of treatment failure. ${ }^{6}$ And it can also cause many side effects, such as allergic reactions, myalgia and neutropenia, etc. ${ }^{7,8}$ Therefore, improving its water solubility and bioavailability and reducing chemotherapy resistance are very essential to improve the treatment strategy for NPC., 10

Magnetic nanoparticles have received tremendous attention in diverse fields because of their unique nanoscale physicochemical properties and the potential for a wide range of applications. ${ }^{11,12}$ In particular, superparamagnetic iron oxide nanoparticles (SPIONs) have become one of the most widely studied targeted nanomaterials. The SPIONs open avenues to drug delivery, ${ }^{13,14}$ magnetic resonance imaging (MRI) ${ }^{11,15}$ and cellular-specific targeting, ${ }^{16}$ and prolong the blood circulation. ${ }^{17}$ The SPIONs accumulate in the tumor site via enhanced permeability and retaining (EPR) effect (passive) or via superparamagnetism properties under an external magnetic field (active). ${ }^{18}$ However, these properties vary significantly within tumors and between tumor types, and are not always sufficient to achieve specific targeting. ${ }^{19}$ Therefore, an alternative strategy for drug delivery systems based on SPIONs is the couple of these systems with targeting segments, such as folic acid (FA), ${ }^{20,21}$ transferrin $(\mathrm{Tf})^{22}$ and antibodies $^{23}$ to improve their targeting performance.

FA is a ligand with a high affinity for the folate receptor (FAR) and is very useful for cancer targeting since many cancer cells overexpress FAR. ${ }^{24}$ FA is its possible conjugation with a number of nanotechnology platforms ${ }^{25}$ and targets drug carriers. ${ }^{24}$ Therefore, FA is used for the drug delivery to increase the efficacy, safety profiles and easy modify, and decrease immunogenicity. ${ }^{20,24,26}$

Recently, lots of studies have reported that the low solubility of PTX can be solved by constructing nanodelivery systems, such as nanoparticles, ${ }^{27}$ liposomes ${ }^{28,29}$ and nanoemulsions. ${ }^{30}$ The desirable nanoparticles should maintain stability in body circulation, highly accumulate in tumor tissues, specifically recognize the targeted cells, and accelerate intracellular drug release to maximize the therapeutic efficacy and minimize the adverse drug reaction. ${ }^{13}$ However, some magnetic nanodrug delivery systems exhibit cumbersome pre-drug preparation steps, expensive reagents, few in vivo distribution studies and low drug loading capacity. Therefore, we have focused on developing a targeting drug delivery system loaded with PTX.

In our work, the 2'-hydroxyl group of PTX was reacted with succinic anhydride to obtain the prodrug of PTX (SPTX). Then, carboxylic group of the succinyl spacer was coupled to FA@PEG/PEI-SPIONs to obtain SPTX@FA@PEG/PEI-SPIONs via solvent volatilization and hydrogen bond adsorption and optimized the formulation by response surface method (RSM). The prepared nano-formulations were characterized and drug entrapment efficiency was calculated. Moreover, the toxicity on nasopharyngeal carcinoma cells and tissue drug distribution of the SPTX@FA@PEG/PEI-SPIONs encapsulated were examined.

\section{Materials and Methods Materials}

PTX was obtained from Huiang biochemical Co., Ltd (Guilin, China). FA was provided by Sinopharm Chemical Reagent (Shanghai, China). Iron (III) acetylacetonate $\left(\mathrm{Fe}(\mathrm{acac})_{3}, 98 \%\right)$ was purchased from Tokyo Kasei Kogyo Co., Ltd. (Tokyo, Japan). 1-(3-Dimethylaminopropyl)3-ethyl-carbodiimide hydrochloride (EDC, 97\%) and N-hydroxysulfosuccinimide sodium salt (sulfo-NHS, 98\%) were provided by Adamas Reagent (Shanghai, China). 4-Dimethylaminopyridine (DMAP), Succinic anhydride and Poly(ethyleneimine) (PEI, $\mathrm{M}_{\mathrm{w}}=1800$ ) were obtained from Aladdin Industrial. Polyethylene glycol (PEG, $\mathrm{M}_{\mathrm{w}}=1000$ ) and other reagents were purchased from Xilong Chemistry Co., Ltd. (Shantou, China). All reagents were used without further purification.

\section{Cell Lines and Animals}

Nasopharyngeal carcinoma CNE-1 cell lines were procured from Xiangya Medical College of South Central University (Changsha, China) ${ }^{31}$ and HNE-1 cells were procured from Fuheng Biology Co., Ltd (Shanghai, China). The use of the cell lines has been approved by the Research Ethics Committee of Guilin Medical University, and the cell has been verified by STR profile. CNE-1 and HNE-1 cells were cultured with RPMI 1640 medium (Gibco, St Louis, MO, USA). All media contained 10\% 
fetal calf serum (FBS, Gibco, St Louis, MO, USA), 100 U/ $\mathrm{mL}$ penicillin and streptomycin (Gibco, St Louis, MO, USA), and the cells were cultured at $37^{\circ} \mathrm{C}$ with $5 \% \mathrm{CO}_{2}$.

Male Sprague Dawley (SD) rats (6-8 weeks old) and Kunming male mice (7-8 weeks old) were provided by the Center of Experimental Animals (Hunan, China, Certificate No. SCXK (Xiang) 2019-0004). Animals were fed a standard rodent diet ad libitum. Animal experiments were approved by the Animal Ethics Committee of Guilin Medical University and carried out in accordance with the Regulations for Animal Experiments and Guidelines for Ethics as defined by the Guilin Medical University (Guangxi, China).

\section{Preparation of SPTX@FA@PEG/ PEI-SPIONs}

\section{Surface Functionalization of PEG/ PEI-SPIONs}

We synthesized PEG/PEI-SPIONs using the polyol pyrolysis method as previously reported. ${ }^{22}$ Briefly, $0.3 \mathrm{~g}$ PEI-1800

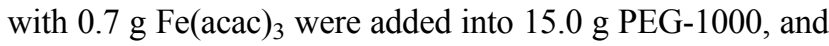
the mixture was heated to $260^{\circ} \mathrm{C}$. For surface functionalization, FA $(0.199 \mathrm{mmol})$ was activated by fully dissolving in $25 \mathrm{~mL}$ of DMSO, and then EDC $(0.398 \mathrm{mmol})$ and sulfoNHS $(0.398 \mathrm{mmol})$ were added and stirred for 3 h. Subsequently, under rapid stirring conditions, PEG/PEISPIONs were slowly added drop-wise to the FA active ester solution, and the mixture was then stirred for 24 h. Afterwards, the sample was isolated by LS magnetic separation column. Finally, the solution was purified with a dialysis bag (MWCO 8000-14000 Da) to remove free folic acid until the dialysate had no UV absorption. FA@PEG/PEI-SPIONs solution was obtained.

\section{Preparation of SPTX@FA@PEG/ PEI-SPIONs by RSM}

PTX was modified using a similar method as described in the previous study. ${ }^{32}$ The detailed synthesis scheme is shown in Supplementary File. The SPTX@FA@PEG/ PEI-SPIONs were prepared using a solvent evaporation and hydrogen bond adsorption technique. Briefly, a certain amount of SPTX was first dissolved in methanol-chloroform (8:2) (as organic phase), then it was added dropwise into FA@PEG/PEI-SPIONs aqueous dispersion (Concentration of $\mathrm{Fe}$ was $1.0 \mathrm{mg} / \mathrm{mL}$ ) (as aqueous phase) with a constant pressure tap funnel. The mixture was then reacted at $24^{\circ} \mathrm{C}$ stirred for $24 \mathrm{~h}$.
Table I Levels of the Factors in RSM Design

\begin{tabular}{|l|c|c|c|c|c|c|}
\hline \multirow{2}{*}{ Variable } & Code & \multicolumn{5}{|c|}{ Levels } \\
\cline { 3 - 7 } & & -1.682 & $-I$ & 0 & $\mathrm{I}$ & $\mathbf{I . 6 8 2}$ \\
\hline $\begin{array}{l}\mathrm{m}_{\text {(SPTX) }} \text { : } \mathrm{m}_{\text {(FA@PEG/PEI- }} \\
\text { SPIONs) }\end{array}$ & $\mathrm{X}_{\mathrm{I}}$ & 0.33 & 0.5 & $\mathrm{I}$ & 2 & 3 \\
\hline $\begin{array}{l}\mathrm{V}_{(\text {(ethanol-chloroform) }} \\
\mathrm{V}_{\text {(H2O) }}\end{array}$ & $\mathrm{X}_{2}$ & 0.2 & 0.33 & 0.5 & $\mathrm{I}$ & 2 \\
\hline $\begin{array}{l}\text { Dropping speed of } \\
\text { SPTX (mL/min) }\end{array}$ & $\mathrm{X}_{3}$ & 0.1 & 0.5 & $\mathrm{I}$ & 2 & 3 \\
\hline
\end{tabular}

On the basis of single factor investigation, the central composite response surface design (central composite design, CCD) was used to select the above three factors: the mass ratio of SPTX and FA@PEG/PEI-SPIONs (m(SPTX): $\left.\mathrm{m}_{\text {(FA@PEG/PEI-SPIONs) }}\right)\left(X_{1}\right)$, the volume ratio of organic phase and aqueous phase $\left(\mathrm{V}_{\text {(ethanol-chloroform): }}\right.$ : $\left.\mathrm{V}_{(\mathrm{H} 2 \mathrm{O})}\right)\left(X_{2}\right)$, and dropping speed of $\operatorname{SPTX}\left(X_{3}\right)$ as the investigation factors (Table 1). The particle size $\left(Y_{1}\right)$ and encapsulation efficiency (EE) $\left(Y_{2}\right)$ were as the evaluation index. The star design of three factors and five levels was carried out. The levels of those independent variables were based on preliminary trials.

\section{Characterization of SPTX@FA@PEG/PEI-SPIONs}

\section{Z-Average Size, Zeta Potential, and Transmission Electron Microscopy}

The average size and Zeta potential of SPTX@FA@PEG/ PEI-SPIONs were determined by Zetasizer Instrument (ZEN 3690, Malvern, USA). All measurements were performed at $25^{\circ} \mathrm{C}$. The morphology of SPTX@FA@PEG/ PEI-SPIONs was observed using a transmission electron microscope (TEM, JEM 2100F, JEOL Ltd., Tokyo, Japan) at $200 \mathrm{kV}$.

\section{Encapsulation Efficiency and Loading Efficiency}

The free SPTX in the SPTX@FA@PEG/PEI-SPIONs dispersion was separated using LS magnetic beads separation column (Miltenyi Biotec, Germany) and the filtrate was collected. To determine EE and loading efficiency (LE) of SPTX, the adsorption of SPTX in the filtrate was calibrated by a standard SPTX solution in the same conditions. The absorption of the filtrate was measured to 
calculate the free SPTX concentration, and the SPTX in the SPTX@FA@PEG/PEI-SPIONs dispersion was determined at $227 \mathrm{~nm}$ using a spectrophotometer (Shimadzu UV3600, Shimadzu Corporation, Kyoto, Japan). The EE and LE contents were calculated using the following formulae:

$\mathrm{EE}(\%)=\left(\mathrm{SPTX}_{\text {total }}-\mathrm{SPTX}_{\text {free }}\right) / \mathrm{SPTX}_{\text {total }} \times 100 \%$

LE $(\%)=\left(\mathrm{SPTX}_{\text {total }}-\mathrm{SPTX}_{\text {free }}\right) / \mathrm{SPTX} @ \mathrm{FA} @ \mathrm{PEG} /$ PEI-SPIONs total $\times 100 \%$

\section{Release of SPTX from SPTX@FA@PEG/ PEI-SPIONs}

The dialysis method was used to analyze the in vitro drug release from the optimal nano-formulation. Briefly, $2 \mathrm{~mL}$ of SPTX@FA@PEG/PEI-SPIONs containing 5 mg SPTX were sealed in dialysis bags (MWCO=8000 14000 Da) and immersed in $50 \mathrm{~mL}$ of PBS solution with $\mathrm{pH} 5.0$, 6.0 or 7.4 , respectively, in an incubator shaker at $37^{\circ} \mathrm{C}$. At predetermined time intervals, $1 \mathrm{~mL}$ of release medium in each vial was withdrawn and replenished with an equal volume of release medium. Finally, the drug concentration in the release medium was determined by the UV spectrophotometer and the cumulative release was calculated.

\section{Stability of SPTX@FA@PEG/ PEI-SPIONs}

The stability of the SPTX@FA@PEG/PEI-SPIONs was assessed in $0.01 \mathrm{~mol} / \mathrm{L}$ PBS solution, in the FBS-containing PBS solution (10\% FBS) and complete cell culture medium ( $10 \% \mathrm{FBS})$ at $37^{\circ} \mathrm{C}$ for $72 \mathrm{~h}$ or at $4^{\circ} \mathrm{C}$ for 28 days. The particle size changes of SPTX@FA@PEG/PEISPIONs were monitored by Zetasizer Instrument over time. The concentration of SPTX in the supernatant was measured by the UV spectrophotometer.

\section{In vitro Cytotoxicity Studies}

The cytotoxic effects of PTX and SPTX were conducted in nasopharyngeal cancer cells (CNE-1 and HNE-1) in vivo using MTT assay. CNE-1 and HNE-1 were cultured in RPMI-1640 medium with $10 \% \mathrm{FBS}, 100 \mathrm{U} / \mathrm{mL}$ penicillin, and $100 \mu \mathrm{g} / \mathrm{mL}$ streptomycin at $37^{\circ} \mathrm{C}$ in a humidified incubator with a $5 \% \mathrm{CO}_{2}$. Exponentially growing $\mathrm{CNE}-1$ and HNE-1 cells were incubated in 96-well plates at the density of $4 \times 10^{3}$ cells per well. After $24 \mathrm{~h}$ incubation, cells were subjected to $200 \mu \mathrm{L}$ fresh medium containing PTX or SPTX $\left(10^{-3}-10^{2} \mu \mathrm{M}\right)$ for $24 \mathrm{~h}$, and PTX or SPTX $\left(1-10^{4}\right.$
$\mathrm{nM}$ ) for $48 \mathrm{~h}$. Cells treated with a medium containing $0.1 \%$ DMSO were used as a control.

The cytotoxicity of blank nanoparticles or drugloaded nanoparticles was also evaluated by MTT assay in nasopharyngeal cancer cells. CNE-1 and HNE-1 cells were seeded in 96-well plates and incubated overnight. Then, the medium was replaced with $200 \mu \mathrm{L}$ fresh medium containing PEG/PEI-SPIONs, PTX, SPTX, FA@PEG/PEI-SPIONs and SPTX@FA@PEG/PEI-SPIONs for 24 h (Fe concentration was $50 \mu \mathrm{g} / \mathrm{mL}$ and PTX or SPTX concentration was $1 \mu \mathrm{M}$ in the medium). Subsequently, $10 \mu \mathrm{L}$ MTT $(5 \mathrm{mg} / \mathrm{mL})$ was added and incubated for another $4 \mathrm{~h}$ in the dark. $100 \mu \mathrm{L}$ DMSO was added to dissolve the formazan crystals. The absorbance at $570 \mathrm{~nm}$ was measured using a BioTek microplate reader (BioTek ELx800, Winooski, Vermont, USA).

\section{In vivo Pharmacokinetics and Biodistribution Study}

The SD rats were randomly divided into three groups $(n=4)$ : PTX group, SPTX group and SPTX@FA@PEG/PEISPIONs group. The rats were intravenously administered PTX, SPTX and SPTX@FA@PEG/PEI-SPIONs at a dose of PTX $(5 \mathrm{mg} / \mathrm{kg})$ or SPTX $(5 \mathrm{mg} / \mathrm{kg})$. Blood was collected into heparinized tubes at different time points $(0.038,0.083$, $0.25,0.5,1,2,4,6,8,10,12 \mathrm{~h}$ ) and centrifuged at $8000 \mathrm{rpm}$ for $10 \mathrm{~min}$ to obtain plasma and stored at $-80^{\circ} \mathrm{C}$.

For the tissue biodistribution study, mice were randomly divided into three groups ( $\mathrm{n}=4$ per group): PTX group, SPTX group and SPTX@FA@PEG/PEI-SPIONs group. Each group was treated with PTX $(5 \mathrm{mg} / \mathrm{kg}$ ) or SPTX $(5 \mathrm{mg} / \mathrm{kg})$ through the tail vein. Mice were euthanized at 1, 4 and 12 $\mathrm{h}$ post injection and different tissues including heart, liver, spleen, lung and kidney were harvested and weighted. Meanwhile, tissue samples were homogenized with tissue homogenizer after the addition of three times the weight of physiologic saline. The concentrations of PTX or SPTX were determined by the high-performance liquid chromatography (HPLC, Agilent 1290 Infinity II, USA) method.

\section{Statistical Analysis}

Statistical significance was measured using the one-way analysis of variance (ANOVA) and Student's $t$-tests, as appropriate. All values were expressed as the mean \pm standard deviation. Values with $p<0.05$ were regarded as significantly 
different. Pharmacokinetic parameters were calculated using the compartmental analysis tool of DAS 2.0.

\section{Results and Discussion Optimization of SPTX@FA@PEG/ PEI-SPIONs by RSM}

SPTX was synthesized by the modified method of Liu et al. ${ }^{32}$ It was confirmed by the HPLC and 1H-NMR analysis that the hydroxyl group of 2 position of PTX was reacted with succinic anhydride. The scheme of the synthesis is illustrated in Figure S1. The retention time was $6.14 \mathrm{~min}$ for PTX and 4.22 min for SPTX (Figure S2) indicating that the chemical structure of the substituent moiety played an important role in the polarity of PTX. SPTX exhibited to be more polar than PTX because of the polar carboxyl moiety. The characteristic peaks of SPTX were found at $2.55 \mathrm{ppm}$ to $2.76 \mathrm{ppm}$ (HOOC-CH2CH2-COO). Nevertheless, the methylene peak of succinic anhydride appeared at $2.57 \mathrm{ppm}$ (Figure S3). The above results indicate that we had successfully modified the structure of PTX and successfully introduced carboxyl (-COOH) in its 2'-OH site.

The water-dispersible SPTX@FA@PEG/PEI-SPIONs conjugates were successfully prepared as illustrated in Figure 1. Firstly, FA was activated by EDC/NHS method, then SPTX was added, and the reaction was carried out by solvent volatilization and hydrogen bond adsorption. Finally, SPTX was attached to FA modified nanoparticles (FA@PEG/PEI-SPIONs), and SPTX conjugates were obtained (SPTX@FA@PEG/PEI-SPIONs).
In RSM design, the effects of three factors on the particle size (Figure 2A1-A3) and EE (Figure 2B1-B3) of SPTX@FA@PEG/PEI-SPIONs were investigated. According to the particle size of SPTX@FA@PEG/PEISPIONs was less than $200 \mathrm{~nm}$ and the EE was the highest, the optimal preparation process of SPTX@FA@PEG/PEISPIONs was determined by Design expert 10.0.7. The 3D plots were based on the function of two variables, with the other variable being at its optimum level. Figure 2A1 and B1 represented the interaction between $\mathrm{m}_{\text {(SPTX) }}: \mathrm{m}_{\text {(FA@PEG/PEI- }}$ SPIONs) and $\mathrm{V}_{\text {(ethanol-chloroform) }} \mathrm{V}_{(\mathrm{H} 2 \mathrm{O})}$. The lower and higher levels of both $\mathrm{m}_{\text {(SPTX) }}: \mathrm{m}_{\text {(FA@PEG/PEI-SPIONs) }}$ and $\mathrm{V}_{\text {(ethanol-chloro- }}$ form) $: \mathrm{V}_{(\mathrm{H} 2 \mathrm{O})}$ did not result in higher $\mathrm{EE}$, but the particle size was increasing. Figure $2 \mathrm{~A} 2$ and $\mathrm{B} 2$ showed the 3D plot corresponding to $\mathrm{m}_{(\mathrm{SPTX})}$ : $\mathrm{m}_{\text {(FA@PEG/PEI-SPIONs) }}$ and dropping speed of SPTX. As shown in Figure 2A3 and B3, the response plot was elliptical, suggestion interaction between them with optimum particle size. Based on the analysis of the data in Figure 2, the optimal preparation process was $\mathrm{m}_{\text {(SPTX) }}$ : $\mathrm{m}$ (FA@PEG/PEI-SPIONs) $=1, \mathrm{~V}_{\text {(ethanol-chloroform) }}: \mathrm{V}_{(\mathrm{H} 2 \mathrm{O})}=1$, dropping speed of SPTX: $1 \mathrm{~mL} / \mathrm{min}$

Three samples were prepared in parallel according to the optimal process. As shown in Table 2, SPTX@FA@PEG/PEI-SPIONs exhibited an intensity diameter of around $178 \mathrm{~nm}$ (predicted value was $169.7 \mathrm{~nm}$ $(\mathrm{RSD}=4.9 \%)$ ) and Zeta potential of around $-10.8 \mathrm{mV}$. The results indicate that the mathematical model is reliable, predictable and relatively stable Besides, the results show that the EE and LE of SPTX@FA@PEG/PEI-SPIONs are around $81.1 \%$ and $14.8 \%$, respectively.

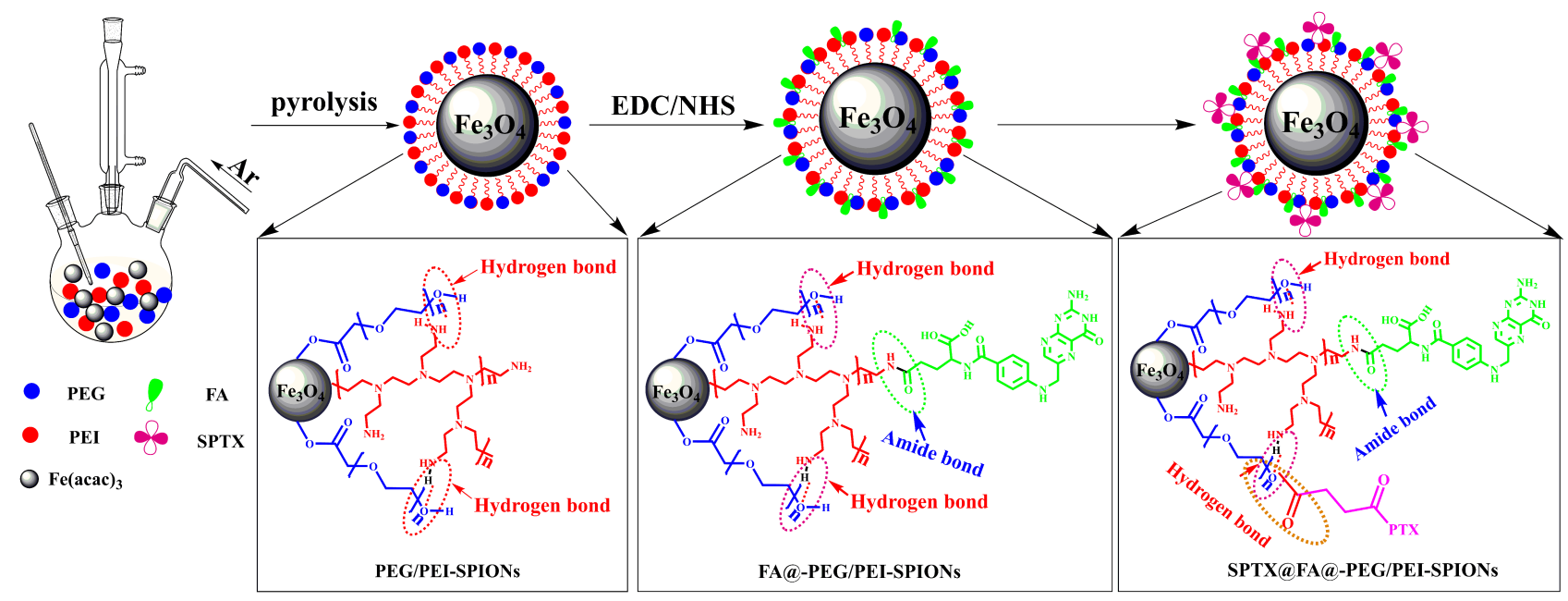

Figure I Synthesis schematic of SPTX@FA@PEG/PEI-SPIONs.

Abbreviations: PEG, polyethylene glycol; PEI, polyethyleneimine; FA, folic acid; SPTX, 2'-succinate paclitaxel. 
A1

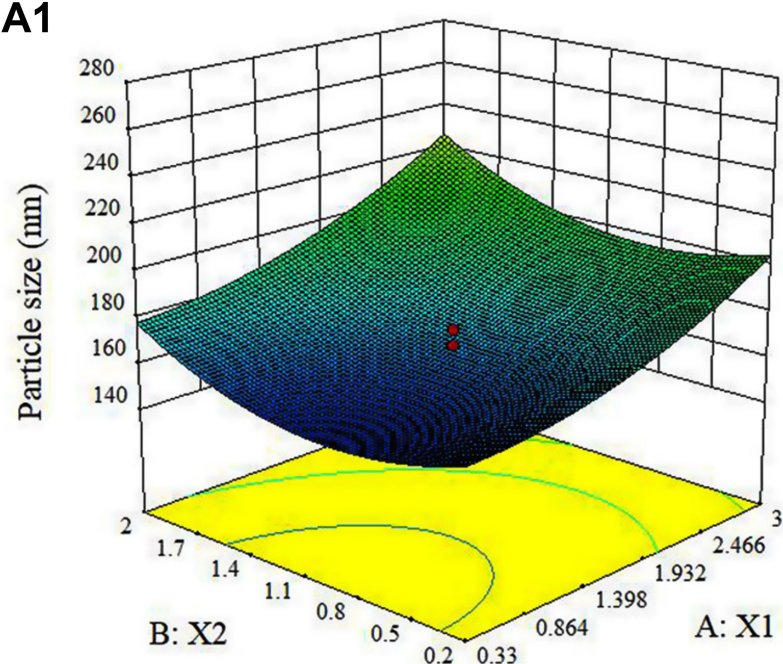

A2

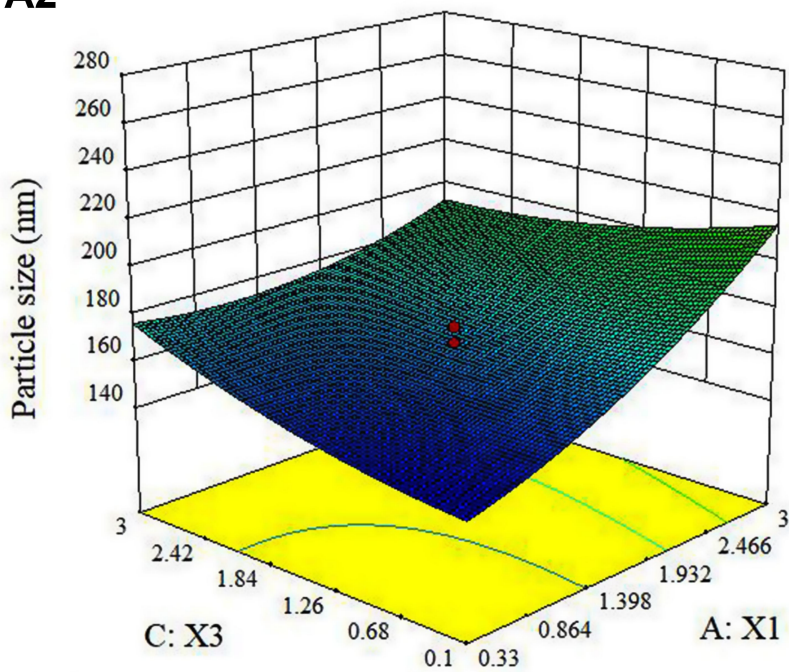

A3

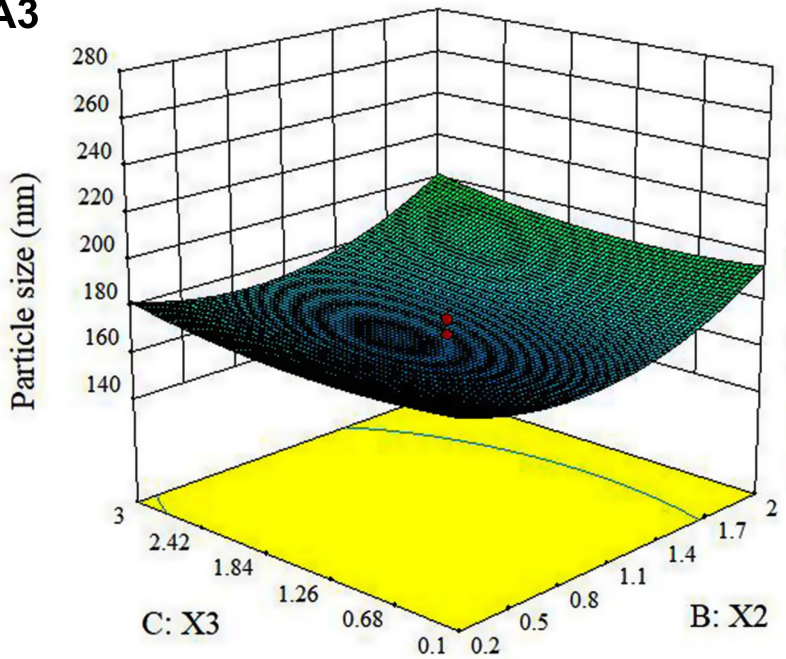

B1

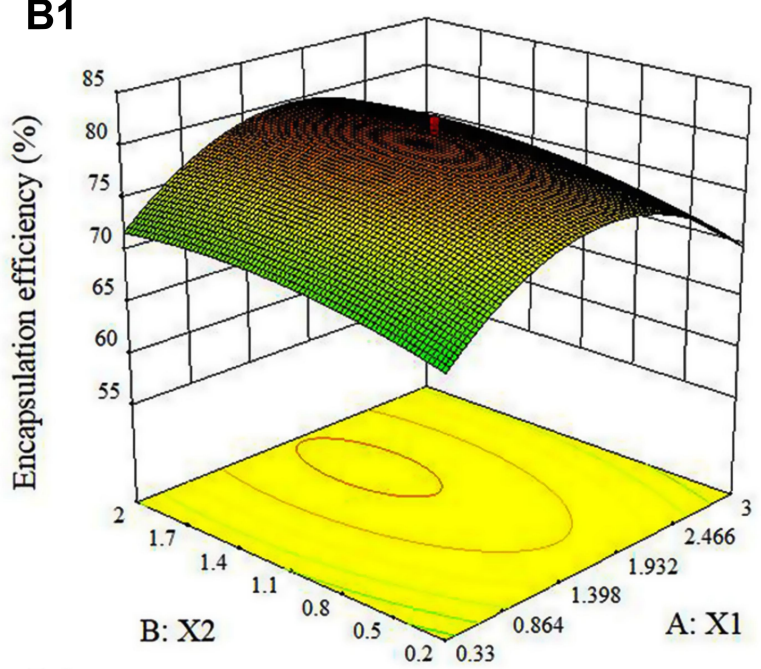

B2

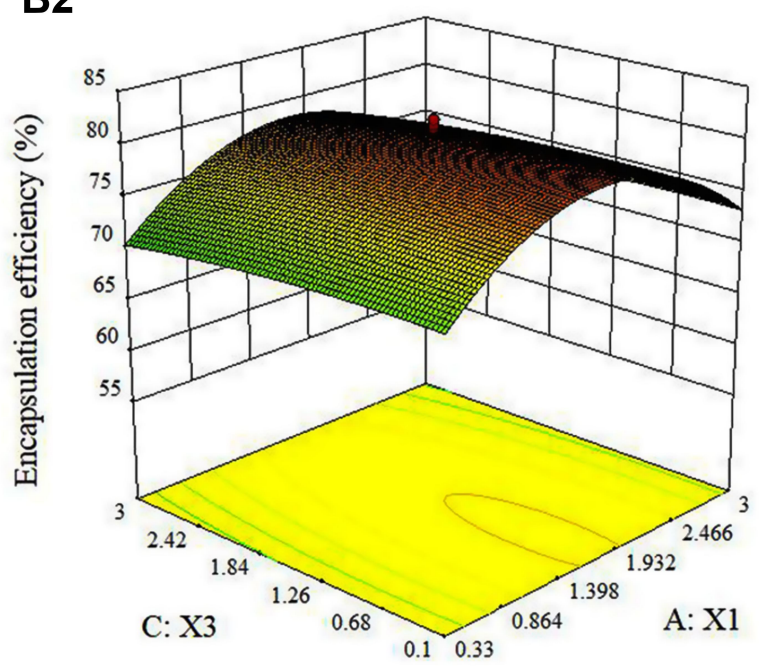

B3

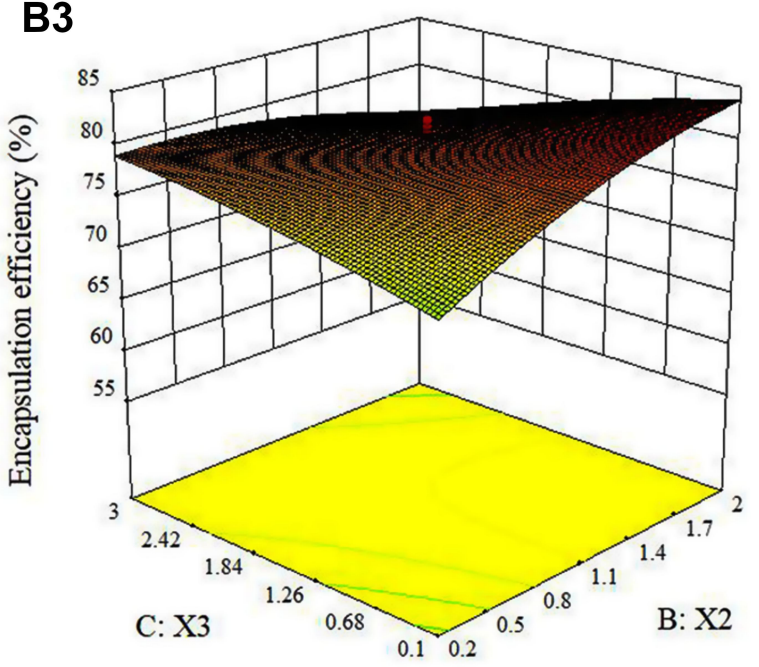

Figure 2 3D response surface diagram for particle size (AI-A3) and EE (BI-B3) of SPTX@FA@PEG/PEI-SPIONs showing interaction between $\left(\mathbf{A} \mathbf{I}\right.$ and $\mathbf{B}$ I) $\mathrm{X}_{\mathbf{I}}$ and $\mathrm{X}_{2}$; (A2 and B2) $X_{1}$ and $X_{3} ;\left(\right.$ A3 and B3) $X_{2}$ and $X_{3}$.

Abbreviations: $\mathrm{m}_{(\mathrm{SPTX})}, \mathrm{m}_{(\mathrm{FA} @ \mathrm{PEG} / \mathrm{PEI}-\mathrm{SPIONs})}\left(\mathrm{X}_{1}\right)$; $\mathrm{V}_{(\text {ethanol-chloroform) }}, \mathrm{V}_{(\mathrm{H} 2 \mathrm{O})}\left(\mathrm{X}_{2}\right)$, dropping speed of SPTX $\left(\mathrm{X}_{3}\right)$. 
Table 2 Results of Certification Optimization

\begin{tabular}{|l|c|c|c|c|}
\hline Number & $\begin{array}{c}\text { Particle } \\
\text { Size (nm) }\end{array}$ & $\begin{array}{c}\text { Zeta } \\
\text { Potential } \\
(\mathbf{m V})\end{array}$ & $\begin{array}{c}\text { EE of } \\
\text { SPTX } \\
(\%)\end{array}$ & $\begin{array}{c}\text { LE of } \\
\text { SPTX } \\
(\%)\end{array}$ \\
\hline 1 & 174.5 & -10.24 & 81.3 & 13.6 \\
\hline 2 & 180.3 & -11.62 & 79.6 & 16.4 \\
\hline 3 & 177.4 & -10.64 & 82.3 & 14.3 \\
\hline $\begin{array}{l}\text { Mean } \pm \\
\text { SD }\end{array}$ & $\begin{array}{c}178.1 \pm \\
3.12\end{array}$ & $-10.8 \pm 0.71$ & $81.1 \pm$ & $14.8 \pm$ \\
1.66 & 1.46 \\
\hline
\end{tabular}

\section{Characterization of SPTX@FA@PEG/PEI-SPIONs}

The SPTX@FA@PEG/PEI-SPIONs exhibited a Z-averaged size of $179.4 \mathrm{~nm}$ (Figure 3A). The Zeta potential of SPTX@FA@PEG/PEI-SPIONs was significantly lower ( $-10 \mathrm{mV})$ than that of FA@PEG/PEI-SPIONs $(\sim-3.4 \mathrm{mV})$ and PEG/PEI-SPIONs $(\sim+19.5 \mathrm{mV})$, most likely due to the SPTX introduced (Figure 3B). The Zeta potential prevents the agglomeration of particles by using electrical repulsion. TEM examination further revealed the particle size and morphology of the SPTX@FA@PEG/PEI-SPIONs. As shown in Figure 3C1-C3, TEM analysis confirms that SPTX@FA@PEG/PEI-SPIONs were almost spherical in shape with an average size distribution of $13 \mathrm{~nm}$.

UV and FTIR spectra analyses were useful tools to investigate intermolecular interactions, through shifting or broadening of the bands in the spectra. ${ }^{28}$ As shown in Figure 4A, the absorption peak of FA at $280 \mathrm{~nm}$ was the characteristic peak and the absorption peaks of $227 \mathrm{~nm}$ and $206 \mathrm{~nm}$ were the characteristic absorption peaks of SPTX. Due to the special structure of SPTX showed terminal absorption. In Figure 4B, FTIR results showed that there was a new absorption peak at $1564.3 \mathrm{~cm}^{-1}$ that the primary amine $\left(-\mathrm{NH}_{2}\right)$ of FA@PEG/PEI-SPIONs disappears and secondary amine $(-\mathrm{NH})$ appeared during the process of synthesis. In addition, the absorption band between $1646.4 \mathrm{~cm}^{-1}$ and $1733.8 \mathrm{~cm}^{-1}$ allocated ketone carbonyl stretching vibration at $\delta$ (-CONH-). The absorption peak $1250.1 \mathrm{~cm}-1$ was related to tensile vibration at $\delta(\mathrm{C}=\mathrm{O}){ }^{28}$ The absorption peaks of SPTX were between $3000 \mathrm{~cm}^{-1}$ to $3250 \mathrm{~cm}^{-1}$ of which were related to tensile vibration at $\delta(-\mathrm{NH})$. The absorption
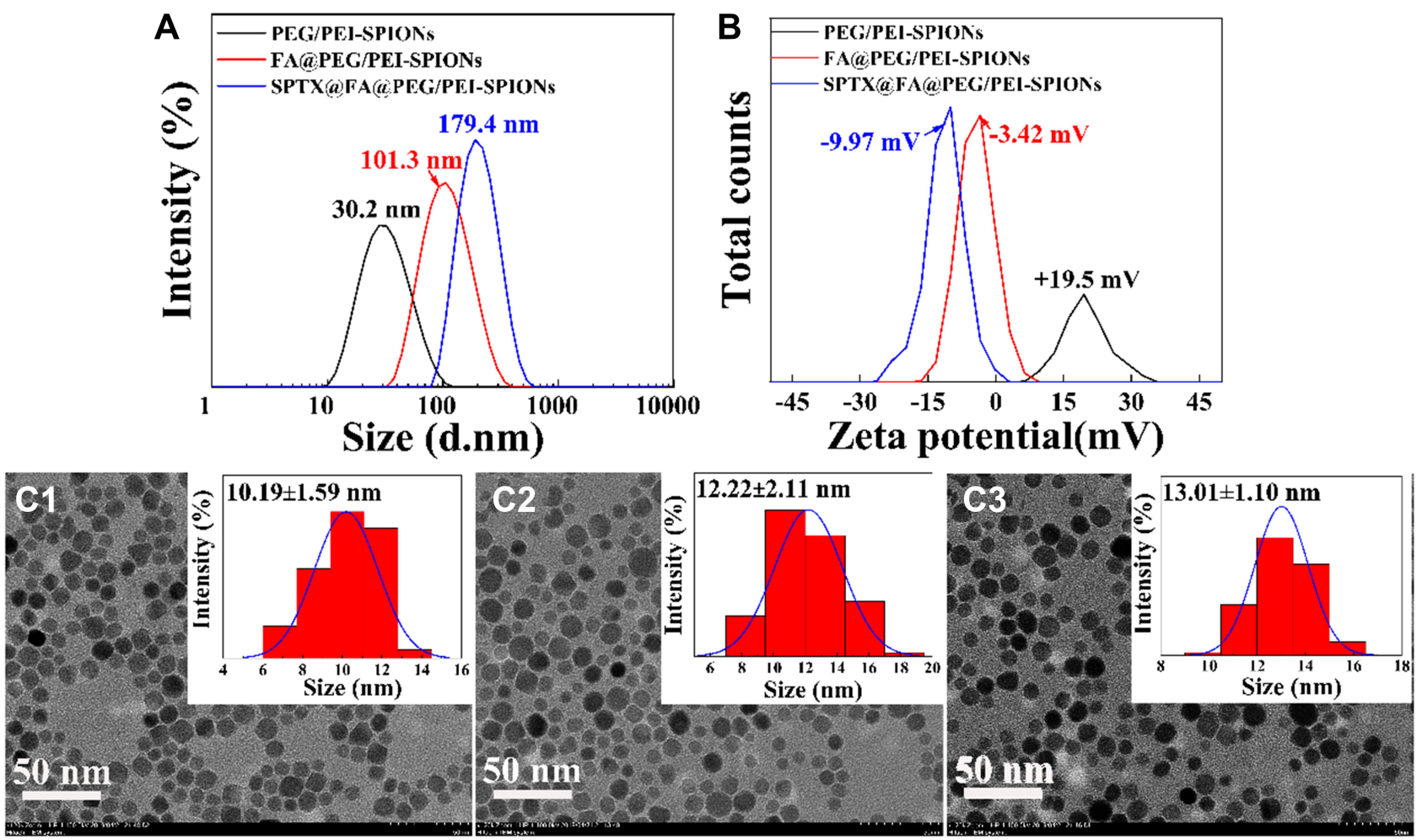

Figure 3 Particles size (A), zeta potential (B), TEM images and size distributions of PEG/PEI-SPIONs (CI), FA@PEG/PEI-SPIONs (C2), SPTX@FA@PEG/PEI-SPIONs (C3) under optimal conditions. 

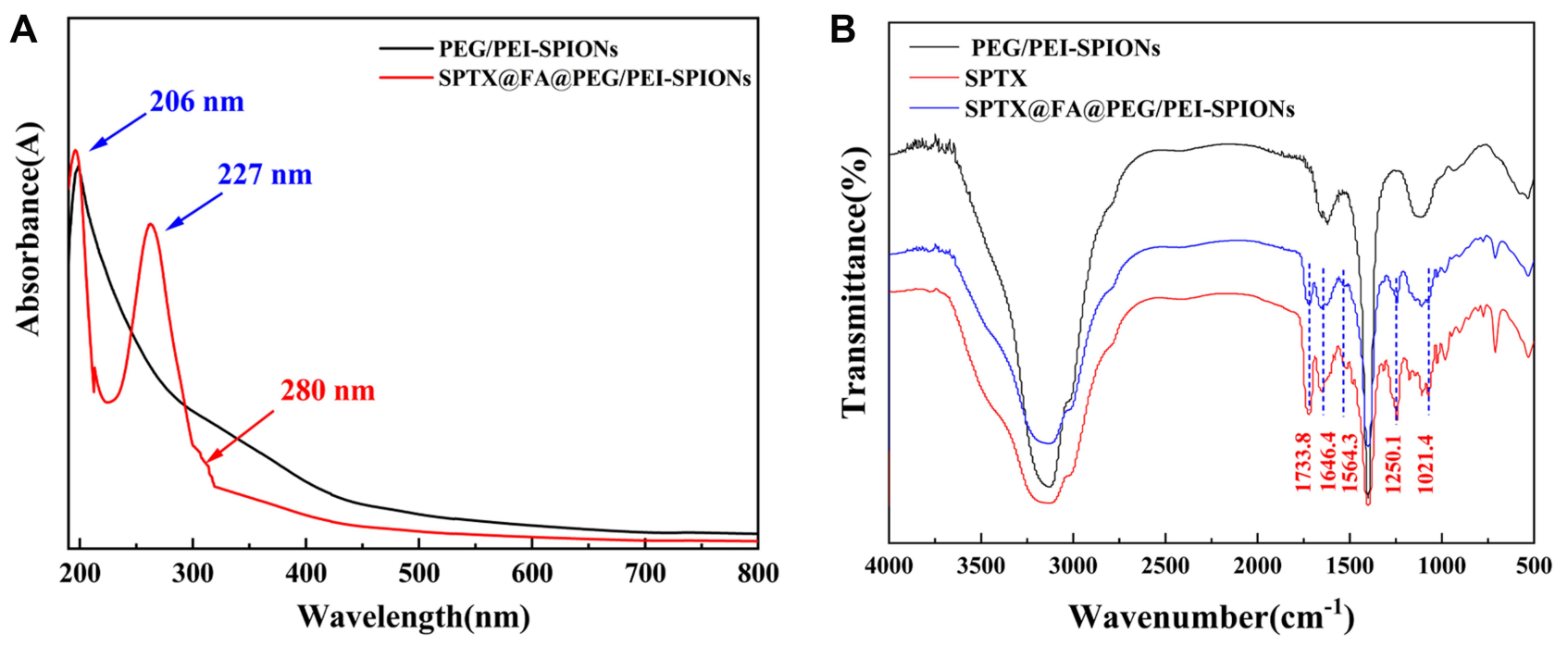

Figure 4 (A) The ultraviolet (UV) and (B) Fourier transform infrared (FTIR) spectra of SPTX@FA@PEG/PEI-SPIONs.

peaks may indicate SPTX loaded FA@PEG/PEISPIONs.

\section{Stability Study}

In order to evaluate the stability of SPTX@FA@PEG/PEISPIONs in vitro, the nanoparticles were incubated with $0.01 \mathrm{~mol} / \mathrm{L}$ PBS solution, in the FBS-containing PBS solution (10\% FBS) and complete cell culture medium $(10 \% \mathrm{FBS})$ at $37^{\circ} \mathrm{C}$ for $72 \mathrm{~h}$ or at $4^{\circ} \mathrm{C}$ for 28 days (Figure 5). As shown in Figure 5A, the particle size of SPTX@FA@PEG/PEI-SPIONs was slightly increased in these media up to $72 \mathrm{~h}$ at $37^{\circ} \mathrm{C}$ (Figure 5A1), indicating that aggregation occurred. But the particle size was relatively stable at $4^{\circ} \mathrm{C}$ for 28 days (Figure $5 \mathrm{~A} 2$ ), indicated that no aggregation occurred. More importantly, when the experimental temperature adjusted to be $37^{\circ} \mathrm{C}$, simulating in vivo environment during blood circulation, the remnant drug percentage was still $>80 \%$ within 48 h (Figure 5B1). In addition, the drug percentage in SPTX@FA@PEG/PEISPIONs remained above $90 \%$ at $4^{\circ} \mathrm{C}$ for up to 7 days. However, the drug content decreased to about $85 \%$ after 7 days (Figure 5B2). The high serum stability of SPTX@FA@PEG/PEI-SPIONs might be attributed to the ideal amphiphilic balance of the drug-loaded assemblies. ${ }^{33}$

\section{In vitro Drug Release}

Next, the in vitro drug release from SPTX@FA@PEG/ PEI-SPIONs in $\mathrm{pH}$ 7.3, 6.0 and 5.8 PBS solutions were studied. SPTX was slowly released within $72 \mathrm{~h}$ at $\mathrm{pH} 7.3$ without an apparent burst release (Figure 6), which indicates that the SPTX@FA@PEG/PEI-SPIONs remain stable with reduced drug leakage during blood circulation. However, when the $\mathrm{pH}$ was 5.0, the drug cumulative released rate from the SPTX@FA@PEG/PEI-SPIONs by SPTX reached more than $82 \%$ of the total drug loading after $48 \mathrm{~h}$. The main reason for this phenomenon was that the electrostatic interaction and hydrogen bonding between coating polymers and SPTX are broken because of the protonation effect at a low $\mathrm{pH}$ value.

\section{In vitro Cytotoxicity Assays}

The cytotoxicity of PTX and SPTX was compared with NPC cells CNE-1 and HNE-1 (Figure 7A and B). At $24 \mathrm{~h}$, the $\mathrm{IC}_{50}$ value for CNE-1 cells for PTX and SPTX were 1.943 and $1.647 \mu \mathrm{M}$. The IC50 values for HNE-1 cells for PTX and SPTX were 3.988 and $3.632 \mu \mathrm{M}$ (Figure 7A). The study indicated that PTX and SPTX were toxic to CNE-1 at a lower concentration than to HNE-1. However, after $48 \mathrm{~h}$ of treatment, the toxicity of PTX or SPTX to CNE-1 cells was significantly reduced (Figure 7B), indicated that HNE-1 was more sensitive to drugs than CNE-1 at $48 \mathrm{~h}$.

The cytotoxicity of the PEG/PEI-SPIONs and FA@PEG/PEI-SPIONs were qualitatively determined using the colorimetric MTT assay on CNE-1 and HNE-1 cell lines. The cells were incubated with $\mathrm{Fe}$ concentration of $20-200 \mu \mathrm{g} / \mathrm{mL}$ of PEG/PEI-SPIONs and FA@PEG/PEI-SPIONs for $24 \mathrm{~h}$. As shown in Figure 7C, after 24-h incubation with PEG/PEISPIONs, the viability of CNE-1 and HNE-1 were still $>80 \%$ at all $\mathrm{Fe}$ incubation concentrations. The FA@PEG/PEI-SPIONs showed no significant cell 
A1

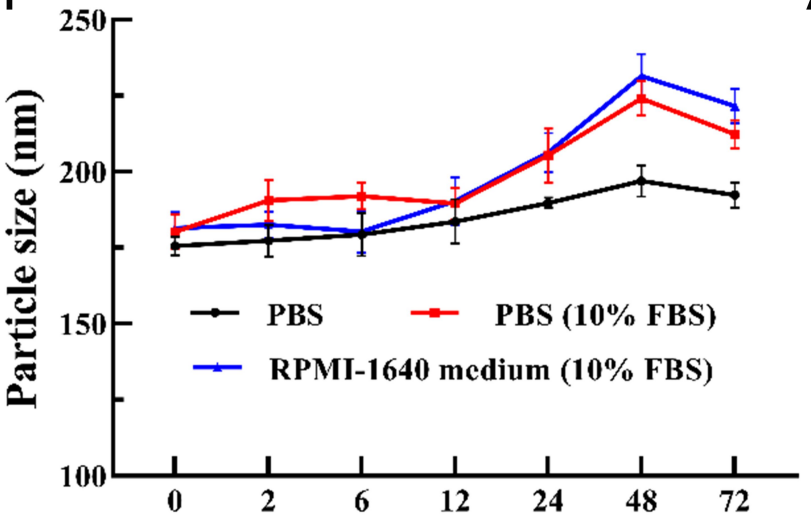

B1

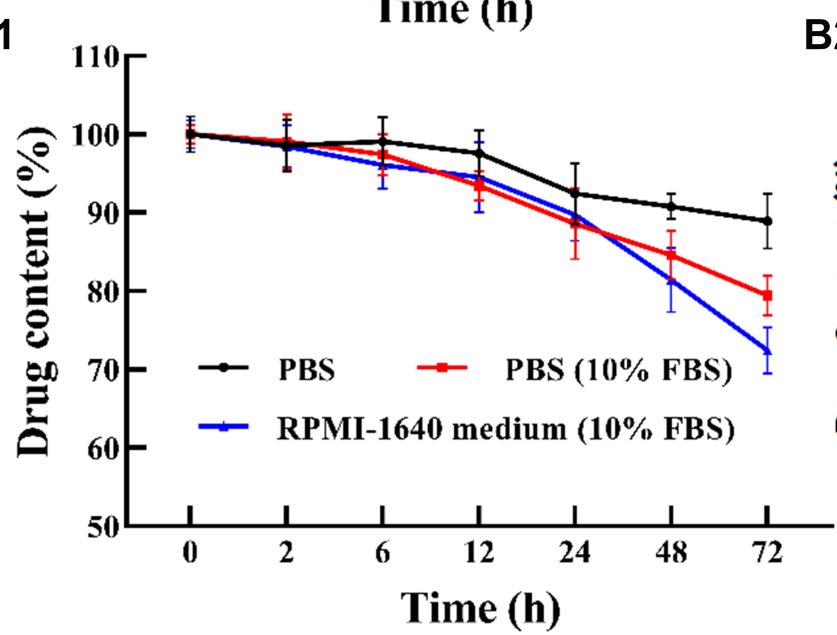

A2

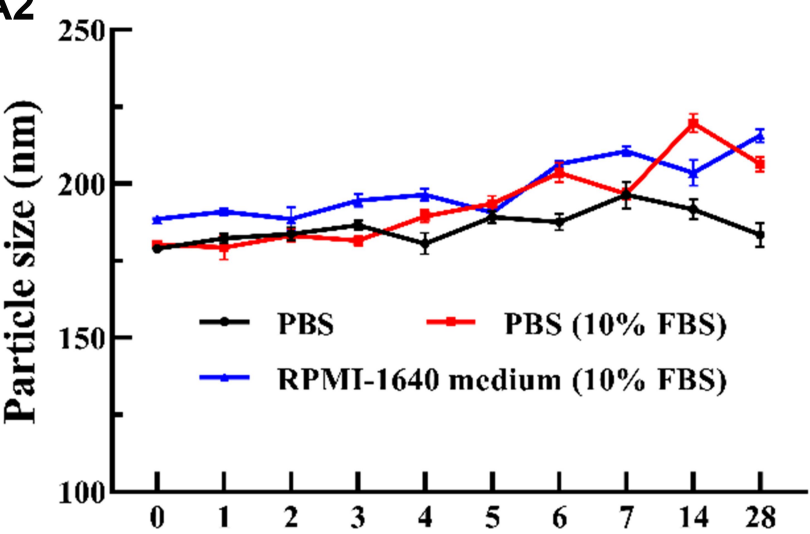

B2

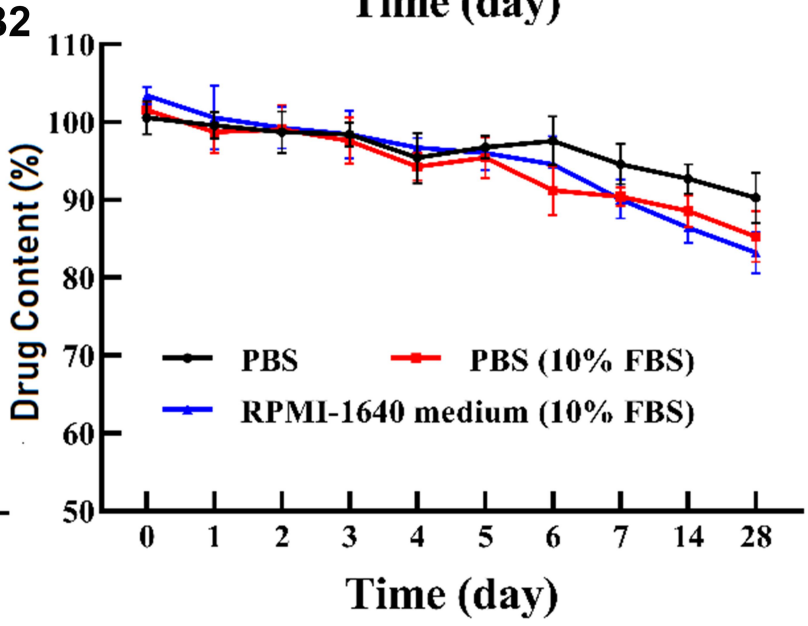

Figure 5 (A) The stability and (B) drug content of SPTX@FA@PEG/PEI-SPIONs in 0.0I mol/L PBS solution, FBS-containing PBS solution (I0\% FBS) and complete cell culture medium (I0\% FBS) at $37^{\circ} \mathrm{C}$ for $72 \mathrm{~h}(\mathbf{A I}$ and $\mathbf{B I})$ or at $4^{\circ} \mathrm{C}$ for 28 days (A2 and B2). Data were represented as mean $\pm \mathrm{SD}$ ( $\mathrm{n}=3$ ).

inhibition on CNE-1 even at the highest concentration of $200 \mu \mathrm{g} / \mathrm{mL}$, but with a concentration $>100 \mu \mathrm{g} / \mathrm{mL}$ had $20 \%$ inhibition on HNE-1 cells (Figure 7D). So PEG/

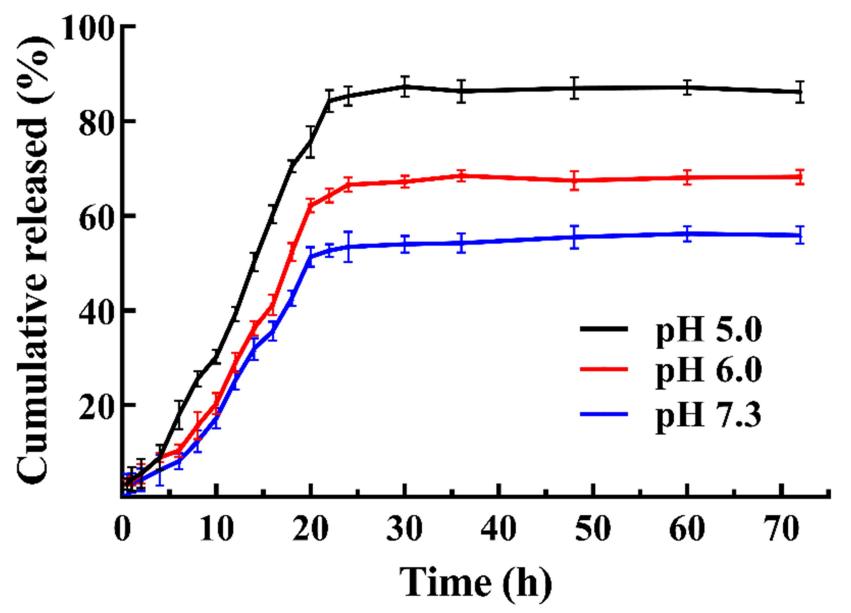

Figure 6 Release profiles of SPTX from SPTX@FA@PEG/PEI-SPIONs at pH 7.3, 6.0 and 5.0 , respectively.
PEI-SPIONs and FA@PEG/PEI-SPIONs possess a reliable level of biocompatibility.

SPTX@FA@PEG/PEI-SPIONs with SPTX concentration of $2.0 \mu \mathrm{M}$ were used to investigate the cytotoxicity of in vitro on NPC cells. The SPTX@FA@PEG/PEI-SPIONs were more cytotoxic than free SPTX or PTX ${ }^{\# \#} P<0.01$, Figure 7E and F), this infers that SPTX@FA@PEG/PEISPIONs enter the cells efficiently via the receptor-mediated endocytosis mechanism, and free SPTX or PTX only diffuse passively into cells. ${ }^{28}$ It can be noticed that the application of magnetic field (MF) increases the cytotoxicity of SPTX@FA@PEG/PEI-SPIONs possibly due to that the MF increases the contact of SPTX@FA@PEG/PEI-SPIONs with the CNE- 1 and HNE- 1 cells $\left(* P<0.05,{ }^{*} P<0.01\right.$, Figure 7E and F). Moreover, due to the active targeting of FAR increases the number of nanoparticles within FAR positive (FAR+) cancer cells (HNE-1) $\left({ }^{\&} \& p<0.01\right.$, Figure 7E and F), the results suggesting that SPTX@FA@PEG/PEI-SPIONs can improve the cytotoxicity to FAR+ cancer cells. These 

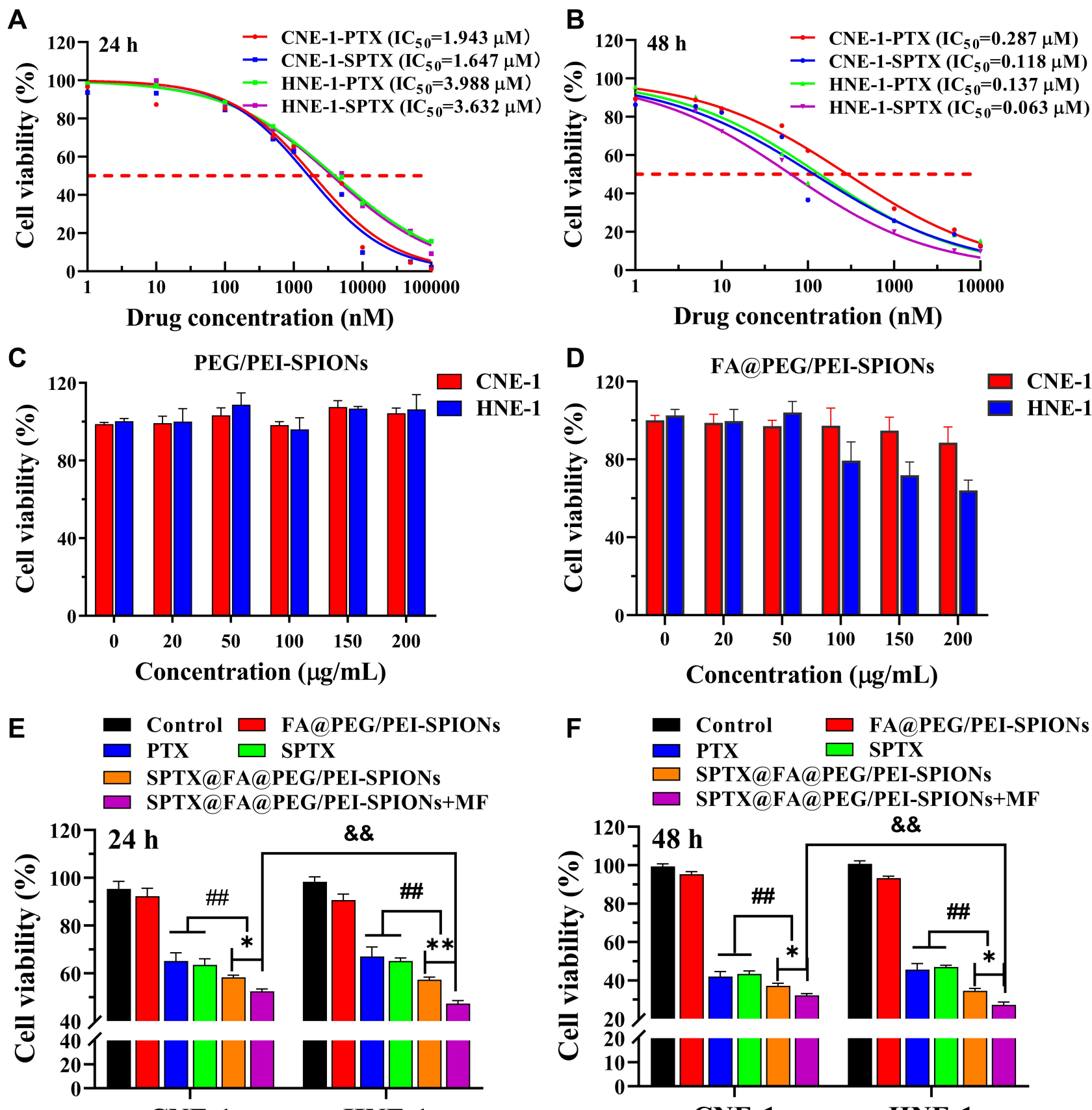

CNE-1 HNE-1

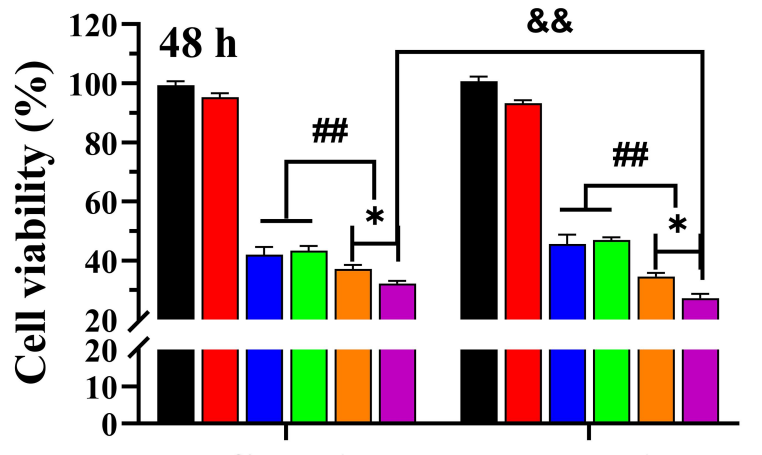

Figure $7 \mathrm{IC}_{50}$ of PTX and SPTX treated with at $24 \mathrm{~h} \mathrm{(A)}$ and $48 \mathrm{~h}$ (B). Concentration-dependent survival curves of CNE-I and HNE-I cells treated by PEG/PEI-SPIONs (C) and FA@PEG/PEI-SPIONs (D) for $24 \mathrm{~h}$. In vitro cytotoxicity of SPTX@FA@PEG/PEI-SPIONs against CNE-I and HNE-I cells after treatment for $24 \mathrm{~h}$ (E) or 48 h (F). Data were expressed as means \pm SD $(n=3) . * P<0.05$, $* * P<0.01 ;{ }^{\#} P<0.01 ;{ }^{2 \&} P<0.01$.

results showed the increased growth inhibition efficiency of SPTX@FA@PEG/PEI-SPIONs on CNE-1 and HNE-1 cells by FA and the MF applied.

\section{In vivo Pharmacokinetics and}

\section{Biodistribution}

The in vivo pharmacokinetics and biodistribution were analyzed after intravenous injection administrated as free PTX,
SPTX and SPTX@FA@PEG/PEI-SPIONs. The plasma drug concentrations were measured by HPLC after different treatments. The results were shown in Figure 8A. The PTX or SPTX plasma concentration at $2 \mathrm{~h}$ after administration of free PTX or SPTX was close to the quantification limit. However, the SPTX plasma concentration was still detectable at $6 \mathrm{~h}$ after administration of the SPTX@FA@PEG/ PEI-SPIONs. The AUC of SPTX@FA@PEG/PEI-SPIONs 
was significantly increased, the $\mathrm{t}_{(1 / 2)}$ was prolonged from 1.67 to $3.41 \mathrm{~h}$, displaying an increased accumulation and decreased clearance of PTX. Thus, SPTX@FA@PEG/PEISPIONs not only achieved higher drug blood levels and longer circulation time in rats but also effectively reduced the in vivo clearance rate.

The concentration of PTX and SPTX in different tissues were presented at three predetermined points $(1,4$ and $12 \mathrm{~h}$ ) after intravenous injection administration of $5 \mathrm{mg} / \mathrm{kg}$ PTX, SPTX or SPTX@FA@PEG/PEI-SPIONs (Figure 8B-D). The biodistribution of SPTX was significantly influenced by the SPTX@FA@PEG/PEI-SPIONs. At the time of $1 \mathrm{~h}$ post administration (Figure 8B), the SPTX concentrations of SPTX@FA@PEG/PEI-SPIONs in the liver $(1.386 \pm 0.227 \mu \mathrm{g} / \mathrm{mL})$, spleen $(0.664 \pm 0.097 \mu \mathrm{g} /$
$\mathrm{mL})$ and kidney $(0.722 \pm 0.107 \mu \mathrm{g} / \mathrm{mL})$ demonstrated the highest values in the whole course, which were 2.00-, 1.35- and 1.60-fold to those of free SPTX group, respectively $\left({ }^{*} P<0.05\right)$. In addition, after $4 \mathrm{~h}$ administration, PTX concentrations of SPTX@FA@PEG/PEI-SPIONs still accumulated higher levels in the liver, kidney and spleen (Figure $8 \mathrm{C},{ }^{*} P<0.05$ ). The accumulation in the liver and spleen of the drug was ascribed to the macrophages and other antigen-presenting cells of the mononuclear phagocyte system (MPS) ${ }^{34}$ and kidney accumulation may be due to the surface functionalization of nanoparticles with folic acid, which is responsible for its affinity towards the kidneys. ${ }^{35}$ However, after 12 $\mathrm{h}$ post-injection, accumulation in the kidney was markedly less. SPTX or PTX concentration was decreased after 12
A

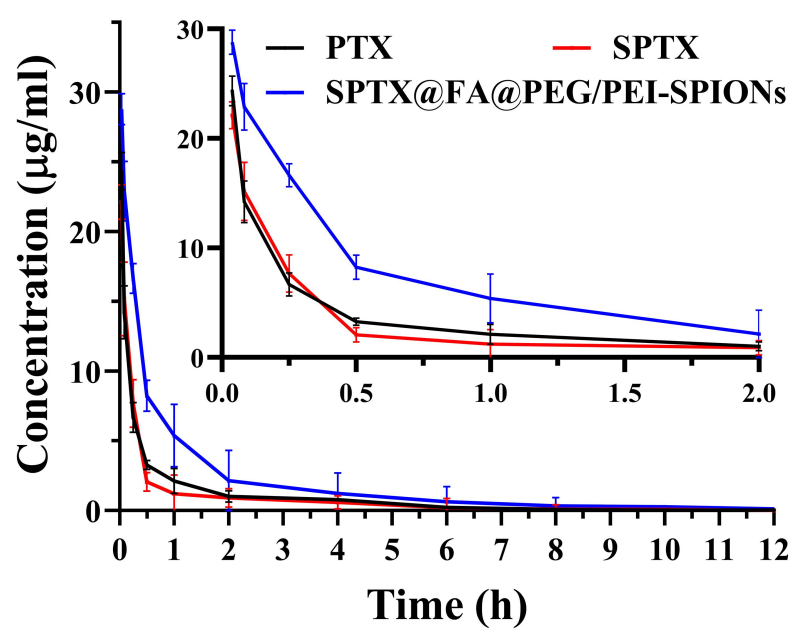

C

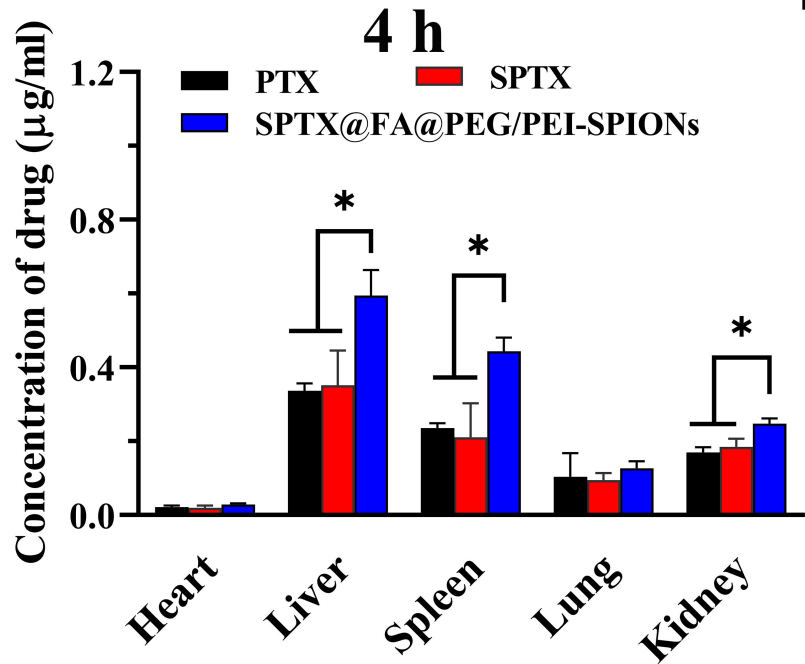

B

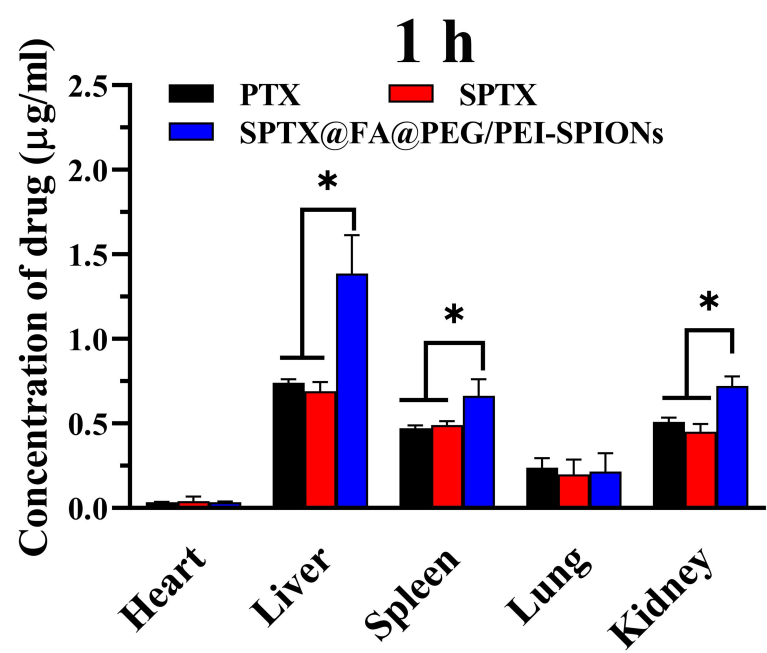

D

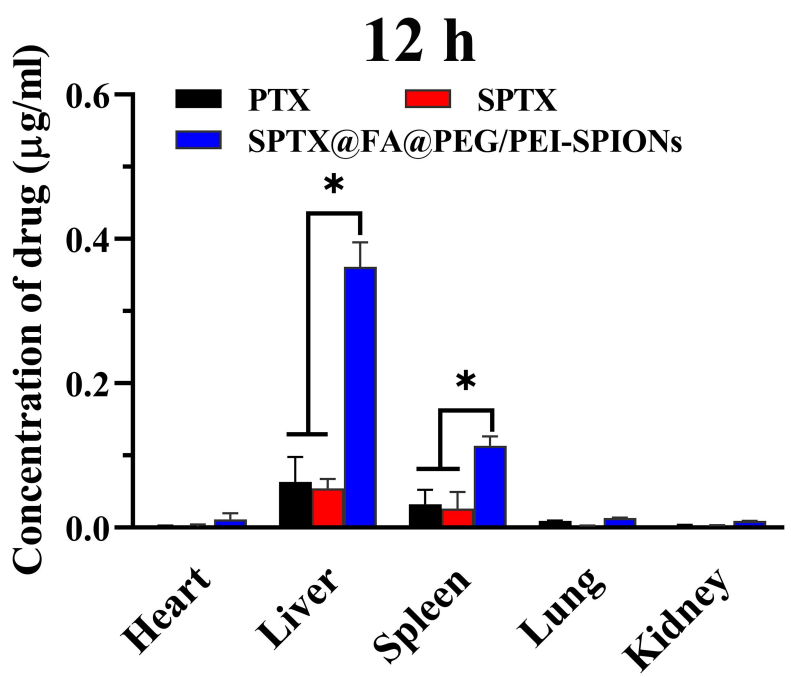

Figure 8 (A) Plasma concentration-time curves of free PTX, SPTX and SPTX@FA@PEG/PEI-SPIONs in vivo, data are expressed as the mean \pm SD ( $=4$ ). Tissue distributions of free PTX, SPTX and SPTX@FA@PEG/PEI-SPIONs at I h (B), 4 h (C) and 12 h (D) post-intravenous injection ( $\mathrm{n}=4$ ), *P<0.05. 
h. This implied that elimination and distribution profiles were similar in all organs (Figure 8D).

\section{Conclusion}

In this study, SPTX@FA@PEG/PEI-SPIONs were synthesized using a solvent evaporation and hydrogen bond adsorption technique. The SPTX@FA@PEG/PEI-SPIONs had a nanoscaled size with a uniform size distribution, exhibited the sustained release, and remained stable in physiological media. The SPTX@FA@PEG/PEI-SPIONs were more efficiently internalized into HNE-1 cells, resulting in the enhancement of cellular uptake of PTX for cytotoxic effects. Importantly, we use PEG/PEI-SPIONs to influence the blood retention half-life of drug molecules and can load target ligands on its surface, thereby enhancing target uptake. The present work utilizes functionalized nanoparticles for targeting folate receptor (FR) positive cancers cell (HNE-1) and helps in targeting the folate receptors present on the kidneys. Though in vivo specificity towards FR and its effect on tumor models in vivo could not be established, but the pharmacokinetics as envisaged in the present design was successfully achieved and could be employed in the future designing of loading hydrophobic drug for improved theranostic applications.

\section{Acknowledgment}

This work was supported by the National Natural Science Foundation of China (No. 81660668), the opening Project of the key Laboratory for early Prevention and treatment of Regional High-incidence tumors in Guangxi (GK2015TKF04) and Guangxi key Laboratory of quality Standards for traditional Chinese Medicine (GZZK-201702).

\section{Disclosure}

The authors state that there are no conflicts of interest in this work.

\section{References}

1. Chen YP, Chan ATC, Le QT, et al. Nasopharyngeal carcinoma. Lancet. 2019;394(10192):64-80. doi:10.1016/S0140-6736(19)30956-0

2. Jing D, Zhou W, Shen L, et al. RIG-I promotes IFN/JAK2 expression and the endoplasmic reticulum stress response to inhibit chemoradiation resistance in nasopharyngeal carcinoma. Cancer Med. 2019;8 (14):6344-6357. doi:10.1002/cam4.2501

3. Xue $\mathrm{C}$, Tian $\mathrm{Y}$, Zhang J, et al. In vitro and in vivo efficacy of afatinib as a single agent or in combination with gemcitabine for the treatment of nasopharyngeal carcinoma. Drug Des Devel Ther. 2016;10:1299-1306. doi:10.2147/DDDT.S94432

4. Zhao MD, Li JQ, Chen FY, et al. Co-delivery of curcumin and paclitaxel by "core-shell" targeting amphiphilic copolymer to reverse resistance in the treatment of ovarian cancer. Int $J$ Nanomedicine. 2019;14:9453-9467. doi:10.2147/IJN.S224579
5. Schettini F, Giuliano M, De Placido S, et al. Nab-paclitaxel for the treatment of triple-negative breast cancer: rationale, clinical data and future perspectives. Cancer Treat Rev. 2016;50:129-141. doi:10.1016/j.ctrv.2016.09.004

6. Hou Y, Zhu Q, Li Z, et al. The FOXM1-ABCC5 axis contributes to paclitaxel resistance in nasopharyngeal carcinoma cells. Cell Death Dis. 2017;8(3):e2659. doi:10.1038/cddis.2017.53

7. Gornstein EL, Schwarz TL. Neurotoxic mechanisms of paclitaxel are local to the distal axon and independent of transport defects. Exp Neurol. 2017;288:153-166. doi:10.1016/j.expneurol.2016.11.015

8. Picard M. Management of Hypersensitivity Reactions to Taxanes. Immunol Allergy Clin North Am. 2017;37(4):679-693. doi:10.1016/ j.iac.2017.07.004

9. Szebeni J, Muggia FM, Alving CR. Complement activation by Cremophor EL as a possible contributor to hypersensitivity to paclitaxel: an in vitro study. J Natl Cancer Inst. 1998;90(4):300-306. doi:10.1093/jnci/90.4.300

10. Du X, Khan AR, Fu M, et al. Current development in the formulations of non-injection administration of paclitaxel. Int $J$ Pharm. 2018;542(1-2):242-252. doi:10.1016/j.ijpharm.2018.03.030

11. Hour FQ, Moghadam AJ, Shakeri-Zadeh A, et al. Magnetic targeted delivery of the SPIONs-labeled mesenchymal stem cells derived from human Wharton's jelly in Alzheimer's rat models. J Control Release. 2020;321:430-441. doi:10.1016/j.jconrel.2020.02.035

12. Zhi D, Yang T, Yang J, et al. Targeting strategies for superparamagnetic iron oxide nanoparticles in cancer therapy. Acta Biomater. 2020;102:13-34. doi:10.1016/j.actbio.2019.11.027

13. Schleich N, Po C, Jacobs D, et al. Comparison of active, passive and magnetic targeting to tumors of multifunctional paclitaxel/ SPIO-loaded nanoparticles for tumor imaging and therapy. J Control Release. 2014;194:82-91. doi:10.1016/j. jconrel.2014.07.059

14. Wallyn J, Anton N, Vandamme TF. Synthesis, principles, and properties of magnetite nanoparticles for in vivo imaging applications-a review. Pharmaceutics. 2019;11(11):601. doi:10.3390/ pharmaceutics 11110601

15. Du C, Liu X, Hu H, et al. Dual-targeting and excretable ultrasmall SPIONs for T1-weighted positive MR imaging of intracranial glioblastoma cells by targeting the lipoprotein receptor-related protein. J Mater Chem B. 2020;8(11):2296-2306. doi:10.1039/c9tb02391g

16. Ni JS, Li Y, Yue W, et al. Nanoparticle-based cell trackers for biomedical applications. Theranostics. 2020;10(4):1923-1947. doi:10.7150/thno.39915

17. Ryu JH, Lee S, Son S, et al. Theranostic nanoparticles for future personalized medicine. J Control Release. 2014;190:477-484. doi:10.1016/j.jconrel.2014.04.027

18. Zou P, Yu Y, Wang YA, et al. Superparamagnetic iron oxide nanotheranostics for targeted cancer cell imaging and $\mathrm{pH}$-dependent intracellular drug release. Mol Pharm. 2010;7(6):1974-1984. doi:10.1021/ mp100273t

19. Guo Y, Wang XY, Chen YL, et al. A light-controllable specific drug delivery nanoplatform for targeted bimodal imaging-guided photothermal/chemo synergistic cancer therapy. Acta Biomater. 2018;80:308-326. doi:10.1016/j.actbio.2018.09.024

20. Montazerabadi A, Beik J, Irajirad R, et al. Folate-modified and curcumin-loaded dendritic magnetite nanocarriers for the targeted thermo-chemotherapy of cancer cells. Artif Cells Nanomed Biotechnol. 2019;47(1):330-340. doi:10.1080/21691401

21. Shaghaghi B, Khoee S, Bonakdar S. Preparation of multifunctional Janus nanoparticles on the basis of SPIONs as targeted drug delivery system. Int $J$ Pharm. 2019;559:1-12. doi:10.1016/j. ijpharm.2019.01.020

22. Wang J, Zhang B, Yang G, et al. Transferrin-conjugated superparamagnetic iron oxide nanoparticles as in vivo magnetic resonance imaging contrast agents. $J$ Nanosci Nanotechnol. 2020;20 (4):2018-2024. doi:10.1166/jnn.2020.17311 
23. Tovar MA, Parkhurst A, Matuczinski E, et al. Synthesis of a superparamagnetic iron oxide based nano-complex for targeted cell death of glioblastoma cells. Nanotechnology. 2019;30 (46):465101. doi:10.1088/1361-6528/ab33d4

24. Huang Y, Mao K, Zhang B, et al. Superparamagnetic iron oxide nanoparticles conjugated with folic acid for dual target-specific drug delivery and MRI in cancer theranostics. Mater Sci Eng C Mater Biol Appl. 2017;70(Pt 1):763-771. doi:10.1016/j.msec.2016.09.052

25. Khademi S, Sarkar S, Shakeri-Zadeh A, et al. Folic acid-cysteamine modified gold nanoparticle as a nanoprobe for targeted computed tomography imaging of cancer cells. Mater Sci Eng C Mater Biol Appl. 2018;89:182-193. doi:10.1016/j.msec.2018.03.015

26. Zeinizade E, Tabei M, Shakeri-Zadeh A, et al. Selective apoptosis induction in cancer cells using folate-conjugated gold nanoparticles and controlling the laser irradiation conditions. Artif Cells Nanomed Biotechnol. 2018;46(sup1):1026-1038. doi:10.1080/21691401.2018.1443116

27. Zheng XC, Ren W, Zhang S, et al. The theranostic efficiency of tumor-specific, $\mathrm{pH}$-responsive, peptide-modified, liposome-containing paclitaxel and superparamagnetic iron oxide nanoparticles. Int J Nanomedicine. 2018;13:1495-1504. doi:10.2147/IJN.S157082

28. Eloy JO, Petrilli R, Topan JF, et al. Co-loaded paclitaxel/rapamycin liposomes: development, characterization and in vitro and in vivo evaluation for breast cancer therapy. Colloids Surf B Biointerfaces. 2016;141:74-82. doi:10.1016/j.colsurfb.2016.01.032

29. Bernabeu E, Cagel M, Lagomarsino E, Moretton M, Chiappetta DA. Paclitaxel: what has been done and the challenges remain ahead. Int J Pharm. 2017;526(1-2):474-495. doi:10.1016/j. ijpharm.2017.05.016
30. Chen L, Chen B, Deng L, et al. An optimized two-vial formulation lipid nanoemulsion of paclitaxel for targeted delivery to tumor. Int J Pharm. 2017;534(1-2):308-315. doi:10.1016/j.ijpharm.2017.10.005

31. Li W, You Y, Zhang X, et al. Amplification of chromosome 8q21-qter associated with the acquired paclitaxel resistance of nasopharyngeal carcinoma cells. Int J Clin Exp Pathol. 2015;8(10):12346-12356.

32. Liu D, Wu W, Chen X, et al. Conjugation of paclitaxel to iron oxide nanoparticles for tumor imaging and therapy. Nanoscale. 2012;4 (7):2306-2310. doi:10.1039/c2nr11918h

33. Huo M, Wang H, Zhang Y, et al. Co-delivery of silybin and paclitaxel by dextran-based nanoparticles for effective anti-tumor treatment through chemotherapy sensitization and microenvironment modulation. J Control Release. 2020;321:198-210. doi:10.1016/j. jconrel.2020.02.017

34. Tang H, Feng X, Zhang T, et al. Stability, pharmacokinetics, biodistribution and safety assessment of folate-conjugated pullulan acetate nanoparticles as cervical cancer targeted drug carriers. J Nanosci Nanotechnol. 2015;15(9):6405-6412. doi:10.1166/jnn.2015.10752

35. Kumar D, Sakhare N, Das S, et al. Development of technetium-99m labeled ultrafine gold nanobioconjugates for targeted imaging of folate receptor positive cancers. Nucl Med Biol. 2020;93:1-10. doi:10.1016/j.nucmedbio.2020.11.001
International Journal of Nanomedicine

\section{Publish your work in this journal}

The International Journal of Nanomedicine is an international, peerreviewed journal focusing on the application of nanotechnology in diagnostics, therapeutics, and drug delivery systems throughout the biomedical field. This journal is indexed on PubMed Central, MedLine, CAS, SciSearch ${ }^{\mathbb{R}}$, Current Contents ${ }^{\mathbb{B}} /$ Clinical Medicine,

\section{Dovepress}

Journal Citation Reports/Science Edition, EMBase, Scopus and the Elsevier Bibliographic databases. The manuscript management system is completely online and includes a very quick and fair peer-review system, which is all easy to use. Visit http://www.dovepress.com/ testimonials.php to read real quotes from published authors. 